\title{
Posible valor pronóstico de la hipertensión arterial en mujeres postmenopáusicas con cáncer de mama. Estudio piloto
}

\author{
J. B. LÓPEZ-SÁEZ, D. QUINTELA SENRA, A. SENRA VARELA \\ Departamento de Medicina. Facultad de Medicina. Universidad de Cádiz. Cádiz
}

\begin{abstract}
POSSIBLE PROGNOSTIC VALUE OF ARTERIAL HYPERTENSION IN POSTMENOPAUSAL WOMEN WITH BREAST CANCER IN COM-
\end{abstract} PLETE CLINICAL REMISSION. PILOT STUDY

\section{RESUMEN}

Fundamento: El objetivo de este trabajo fue determinar la presión arterial (PA) en mujeres postmenopáusicas con cáncer de mama en remisión clínica completa de larga duración.

Material y métodos: Se trata de un estudio piloto, de casos y controles, en los cuales se midieron la presión arterial de 83 mujeres postmenopáusicas, con cáncer de mama histológicamente confirmado en situación de remisión clínica completa de larga duración comparándolo con un grupo control de 70 mujeres sanas postmenopáusicas, de la misma edad. Se calculó el índice de masa corporal (BMI), la superficie corporal, el intervalo de confianza de la media, la correlación entre el BMI y la PA, en ambos grupos (cáncer de mama/mujeres sanas) y las presiones sistólicas y diastólicas.

Resultados: La media de la presión sistólica en las 83 mujeres con cáncer de mama fue de $163 \mathrm{~mm}$ de $\mathrm{Hg}$ (95\% CI 155-171) y en los 70 controles normales fue de $134 \mathrm{~mm}$ de $\mathrm{Hg}$ (95\% CI 129-139). La diferencia entre ambos grupos fue estadísticamente significativa $(\mathrm{p}<0,001)$. La media de la presión diastólica en las mujeres con cáncer fue de 98 mm de $\mathrm{Hg}$ (94-104) y en los controles normales fue de $78 \mathrm{~mm}$ de $\mathrm{Hg}$ (74-82). La diferencia entre ambos grupos fue estadísticamente significativa $(\mathrm{p}<0,001)$.

Conclusiones: La hipertensión arterial, independientemente del BMI y de la duración del intervalo libre de enfermedad, está asociado con una mayor duración de la remisión clínica completa en las mujeres postmenopáusicas con cáncer de mama y consecuentemente con un mejor pronóstico de su enfermedad cancerosa.

PALABRAS CLAVE: Cáncer de mama. Hipertensión arterial. Enzima convertidora de angiotensina.

\begin{abstract}
Background: The objective of this work was to determine the blood pressure of postmenopausal women with breast cancer in complete clini cal remission of long duration.

Material and methods: It in a pilot study of case and controls, in which we meassure the Blood Pressure (BP) of 83 postmenopausal women, with breast cancer histologically confirmed, in complete clinical remission of long duration, recruited by consecutive sampling, to compa re it with that of 70 normal postmenopausal women of the same age used as controls. They ara calculated the body mass index (BMI), the corporal surface, the confidence intervals $(C I)$ of the means, the correlation between the BMI and the BP in both groups (breast cancer patients and normal control) and between the free disease interval and the systolic and diastolic blood pressures.

Results: The mean of the systolic BP in 93 breast cancer patients in complete clinical remission was $163 \mathrm{~mm} \mathrm{Hg}$ (95\% CI 155-171) and in 70 normal controls was $134 \mathrm{~mm} \mathrm{Hg}$ (95\% CI 129-139). The difference bet ween both groups in statistically significant $(p<0.001)$. The mean of the diastolic BP in the breast cancer patient in complete remission it was 98 $\mathrm{mm} \mathrm{Hg} \mathrm{(94-104)} \mathrm{and} \mathrm{in} \mathrm{the} \mathrm{normal} \mathrm{controls} \mathrm{was} 78 \mathrm{~mm} \mathrm{Hg}$ (74-82). The difference between both groups was statistically significant $(p<0.001)$.

Conclusions: This arterial hypertension, independent of the BMI and from the duration of the free disease interval, is associated with a long duration of the complete remission in postmenopausal breast cancer patients and consequently with a good prognostic of this disease.
\end{abstract}

KEY WORDS: Breast cancer. Arterial hypertension. Angiotensin con verting enzyme.

López-Sáez JB, Quintela Senra D, Senra Varela A. Posible valor pronóstico de la hipertensión arterial en mujeres postmenopáusicas con cáncer de mama. Estudio piloto. An Med Interna (Madrid) 2001; 18: 132-135.

\section{INTRODUCCIÓN}

La presión arterial está regulada por una serie de mecanismos entre los cuales resalta por su importancia la acción de la Enzima Conversora de la Angiotensina (ECA), la cual es una enzima proteolítica que transforma la angiotensina I (decapéptido) en angiotensina II (octapéptido) que eleva la presión arterial, mediante un aumento en el tono de la pared vascular arterial. La ECA tiene algunas importantes funciones biológicas en el sistema cardiovascular, revisadas por Dostal et al (1). La ECA forma parte de un complejo sistema, aún no bien comprendido y claramente relacionado con la mitosis celular. Bell y cols. (2) han demostrado que la inhibición del sistema autocrino de la angiotensina con un inhibidor de la ECA o con

Trabajo aceptado: 4 de Septiembre de 2000

Correspondencia: J. Bosco López Sáez. Departamento de Medicina. Facultad de Medicina. Universidad de Cádiz. C/ Dr. Marañón, 3. 11002 Cádiz. 
un antagonista del receptor conduce a un aumento de la expresión del protooncogén c-src; por otra parte, el captopril que es un inhibidor de la ECA inhibe las mitosis en las células tumorales de un cáncer ductal de páncreas del hámster, células que carecen de actividad ECA y renina (3).

En un trabajo previo hemos descrito la elevación por encima de los valores normales de la ECA sérica en los casos de remisión clínica completa de varios tumores malignos y su descenso por debajo de los valores normales en las recaídas tumorales (4). Por otra parte, hemos adquirido la experiencia de observar una mayor frecuencia de hipertensión arterial en la mayoría de los casos de cáncer de mama en remisión clínica completa de larga duración en mujeres postmenopáusicas. Estábamos interesados en comprobar dicha observación y el propósito de este estudio piloto es sometido a comprobación.

El objetivo de este trabajo fue determinar la cuantía de la hipertensión arterial en mujeres postmenopáusicas con cáncer de mama en remisión clínica completa de larga duración, comparativamente con mujeres normales del mismo grupo socioeconómico y de la misma edad.

\section{MATERIAL Y MÉTODO}

Es un estudio piloto de casos y controles, en el cual medimos tres veces la presión arterial de 83 mujeres postmenopáusicas, con cáncer de histológicamente confirmado, en remisión clínica completa de larga duración (>5 años), para compararla con la de 70 mujeres postmenopáusicas normales de la misma edad usadas como controles.

Es un muestreo consecutivo, de enfermas con cáncer de mama previamente operadas y en remisión clínica completa, que están siendo controladas en seguimiento clínico en los Hospitales "Universitario de Puerta del Mar" y "Universitario de Puerto Real" y Hospital de "San Juan de Dios", de Cádiz. Los controles normales también son mujeres postmenopáusicas, generalmente parientes de las enfermas.

Se ha calculado el índice de masa corporal $(\mathrm{IMC})=\mathrm{P} / \mathrm{T}^{2} \mathrm{y}$ la superficie corporal $(\mathrm{S})=\mathrm{T}^{\wedge} 0,725 \times \mathrm{P}^{\wedge} 0,425 \times 71,84 \times 10^{-4}$. La presión arterial (PA), fue medida mediante un esfignomanómetro aneroide en tres ocasiones, separadas cinco minutos y después de un tiempo de reposo de veinte minutos, se tomó con cifra definitiva la media de las tres mediciones previas. Se calculó la correlación mediante el test de Pearson, entre el IMC y la PA en ambos grupos (cáncer de mama y controles normales) y entre el intervalo libre de enfermedad y las presiones sistólica y diastólica. Se compararon las medianas, aplicando el test de la U de Mann-Withney, de las presiones sanguíneas sistólica y diastólica en las enfermas con cáncer de mama en remisión clínica completa y en los controles normales (5) y por último, se calcularon los intervalos de confianza de las medias $(6,7)$.

\section{RESULTADOS}

La media de la presión sistólica en las 83 enfermas de cáncer en remisión clínica completa fue de $163 \mathrm{~mm} \mathrm{Hg}$ con unos intervalos de confianza (IC) para una fiabilidad del $95 \%$ de $145-161$ y en 70 controles normales fue de $134 \mathrm{~mm} \mathrm{Hg}$ (IC de $95 \%, 129-139)$. La diferencia entra ambos grupos fue estadísticamente significativa $(\mathrm{p}<0,001)$.
La media de la presión diastólica en las enfermas de cáncer de mama fue de 98 mm Hg (94-104) y en los controles normales fueron des $78 \mathrm{~mm} \mathrm{Hg}$ (74-82). La diferencia entre ambos grupos fue estadísticamente significativa $(\mathrm{p}<0,001)$.

La media del IMC de las 83 enfermas de cáncer de mama fue de 31 (IC 29-32) y en las 70 mujeres normales fue de 29 (27-30), aplicando el test de la U de Mann-Withney la diferencia fue estadísticamente significativa $(\mathrm{p}<0,05)$ (Tabla I).

\section{TABLA I}

RESUM EN DE LOS RESULTADOS

\begin{tabular}{lcccc}
\hline $\begin{array}{l}\text { Presión } \\
\text { Sistolica }\end{array}$ & N & Media & I.C. del 95\% & Valor de $\mathrm{p}$ \\
\hline $\begin{array}{l}\text { Cáncer de mama } \\
\text { Controles }\end{array}$ & 83 & 163 & $155-171$ & \\
$\begin{array}{l}\text { Presión } \\
\text { Diastólica }\end{array}$ & 70 & 134 & $129-139$ & $\mathrm{P}<0,001$ \\
$\begin{array}{l}\text { Cáncer de mama } \\
\text { Controles }\end{array}$ & 83 & 98 & $94-104$ & $\mathrm{P}<0,001$ \\
$\begin{array}{l}\text { IMC } \\
\text { Cáncer de mama }\end{array}$ & 83 & 78 & $74-82$ & \\
Controles & 70 & 28,9 & $27-30$ & \\
\hline
\end{tabular}

La media de la superficie corporal fue igual (1,67 metros cuadrados) en las enfermas con cáncer de mama y en los controles normales. La correlación entre los valores de la presión

sanguínea sistólica y diastólica y el IMC de las enfermas con cáncer de mama y también en las mujeres normales no fue estadísticamente significativa ( $\mathrm{p}>0,05)$.

El intervalo libre de enfermedad tuvo una media de 7.4 años $(\mathrm{DE}=2,1)$ y no hubo correlación significativa entre el intervalo libre de enfermedad de cada caso y los valores de su presión sistólica o diastólica.

Existen antecedentes claros de hipertensión arterial desde el momento de la mastectomía en 20 casos (24\%); las cuales fueron tratados de su hipertensión arterial. En estos casos los valores de la presión arterial se han elevado. En los restantes casos fue imposible demostrar este antecedente en su historia clínica anterior al desarrollo tumoral.

\section{DISCUSIÓN}

Del presente estudio se deduce que hay una hipertensión arterial asociada con el cáncer de mama en mujeres postmenopáusicas en remisión clínica completa de larga duración.

La hipertensión arterial, es tanto sistólica como diastólica y no se correlaciona con el sobrepeso propio de las enfermas postmenopáusicas con cáncer de mama. La asociación de la hipertensión arterial con las remisiones clínicas prolongadas del cáncer de mama en las mujeres postmenopáusicas, de algún modo implica un buen pronóstico del cáncer de mama, con independencia de cual esa el mecanismo por el cuál actúa como protectora esta hipertensión arterial. 
Es importante resaltar que la cuantía de la hipertensión arterial no se correlaciona con la duración total del intervalo libre de enfermedad, lo cual implicaría que el desarrollo de la hipertensión arterial es un fenómeno al azar o independiente, que marca el estado de la remisión completa, es decir, facilita o favorece el control del tumor.

En un estudio transversal de casos y controles no podemos saber en que modo está la hipertensión arterial relacionada con la más favorable evolución clínica de la enfermedad cancerosa: a) Si la hipertensión arterial es previa a la misma ó b) Si ésta aparece o se agrava con la presencia del tumor maligno.

Pero se puede concluir afirmando que hay una fuerte asociación entre la remisión clínica completa del cáncer en mujeres postmenopáusicas y la hipertensión arterial absoluta o relativa; bien sea por la ventaja que supone tener mejor pronóstico los casos de cáncer con hipertensión arterial previa o si la hipertensión arterial adquirida en el curso evolutivo de la enfermedad forma parte de la reacción organísmica contra el cáncer, sería en este caso una especie de factor de malignant cell dormancy (8).

No hemos incluido en el estudio a enfermas en recaída de su enfermedad por ser más difícil obtener un número suficiente de enfermas aún no tratadas. La mayor parte de los tratamientos quimioterápicos producen hipotensión arterial y es difícil evaluar la hipotensión arterial habitual en estas enfermas en un estudio transversal.

Hay un IMC significativamente más alto en las mujeres con cáncer de mama, pero éste mayor IMC no es la causa de la hipertensión arterial observada en las enfermas; ya que no existe correlación entre ambos valores ni en las enfermas ni en los controles normales (5).

La edad es un factor importante en la hipertensión arterial; pero en nuestros casos no había una diferencia significativa entre la edad de los casos y los controles (9).

La hipertensión arterial no se puede relacionar con el tipo histológico del tumor, ya que si bien todos los casos eran carcinomas ductales infiltrantes, éste es el tipo histológico habitual. Tampoco se puede atribuir a la medicación antitumoral recibida, ya que un buen número de casos fueron operados en edad premenopáusica y recibieron quimioterapia adyuvante tipo CMF y la otra mitad recibieron hormonoterapia con tamoxifeno, sin diferencias sustantivas entre ambos grupos.

Sin embargo, es imposible excluir que estas enfermas hayan tomado el inhibidor de la ECA, captopril, el cual tiene acciones antimitóticas y que este sea el responsable del mejor pronóstico o mayor supervivencia libre de enfermedad en estas enfermas con cáncer de mama (3).

Parece lógico pensar que la hipertensión observada en las enfermas con cáncer de mama está relacionada con la elevación de la ECA. En esta muestra de enfermas no hemos estudiado los valores de la actividad ECA del suero; pero lo hemos hecho en un estudio previo de nuestro grupo y se demostró que la actividad ECA está aumentada comparativamente con los controles normales en las situaciones de remisión clínica completa y disminuye de un modo significativo, por debajo de los valores normales de referencia en las recaídas o fases activas de la enfermedad (4).

La elevación de la actividad ECA del suero de las enfermas con cáncer de mama en remisión clínica comple- ta sería un auténtico marcador de remisión clínica completa y sería el mecanismo de la hipertensión observada por nosotros.

El significado de la hipertensión arterial es difícil de descifrar aún; pero si lo interpretamos de un modo indirecto a través de la elevación de la actividad de la ECA sérica y le son de aplicación los estudios de Bell et al (2) los cuales han demostrado que la inhibición del sistema autocrino de producción de ECA con un inhibidor de la ECA produce un aumento en la remisión del protooncogén c-src, relacionado con las mitosis celulares (2).

Frame et al, demostraron que en los quistes mamarios con bajo riesgo de desarrollar cáncer de mama hay valores altos de ECA en el líquido quístico (30.8 nmoles), mientras en los quistes tapizados por un epitelio apocrino con un alto riesgo de desarrollar cáncer de mama tienen valores muy bajos de actividad ECA (6,1 nmoles), reflejando un efecto protector de la ECA frente al desarrollo de tumores malignos en los quistes mamarios (10).

La distribución en los tejidos periféricos y en el suero de la enzima prolil-oligopeptidasa, que es paralela a la de la ECA, incluso en la patogenia de la hipertensión arterial, disminuye en el suero de las enfermas cancerosas y aumenta en los tejidos tumorales (11). Franko et al, demostraron que hay una correlación inversa entre el desarrollo de fibrosis pulmonar experimental, postradioterapia, en el ratón y la actividad ECA intrínseca del pulmón, implicando un control de la actividad ECA sobre las mitosis de los fibroblastos (12).

Estos estudios de Franko et al (11) y los de Frame et al (12) parecen contradictorios con los de Reddy et al (3) quienes demostraron que los inhibidores de la ECA inhiben las mitosis y la expresión genética en células del cáncer de páncreas.

En todo caso, no podemos aún formular ninguna hipótesis sobre el mecanismo de acción de la ECA en la carcinogénesis ya que resulta difícil de entender que ésta pueda inhibir las mitosis y que los inhibidores de la ECA también. Pudiera crear alguna preocupación a los usuarios de los inhibidores de la ECA la posibilidad de que éstos sean carcinógenos y debemos señalar que en un estudio masivo sobre 47.351 hipertensos, consumidores de inhibidores de la ECA durante un año, se han producido sólo 37 fallecimientos por cáncer, cuando era de esperar una cifra tres veces mayor (13). No podemos descartar en ente caso el efecto protector de la propia hipertensión arterial.

En un estudio previo sobre 512 enfermas con cáncer de mama, hemos descrito la observación, basada en el estudio electrocardiográfico de las enfermas en la primera visita a la consulta de oncología médica, de la existencia de una gran prevalencia de insuficiencia coronaria en las mujeres portadoras de cáncer, tanto en casos pre como postmenopáusicas, comparándolas con los controles normales no tumorales (14) y es sobradamente conocida la relación existente entre insuficiencia coronaria e hipertensión arterial (9).

En un estudio prospectivo a realizar en el futuro pensamos investigar la prevalencia de complicaciones relacionadas con esta hipertensión arterial, la naturaleza de la misma y las posibilidades de emplear la hipertensión arterial como un predictor de recaída. 


\section{Bibliografía}

1. Dostal OF, Booz GW, Baker KM. The cardiac renin-angiotensin system: an overview. In Lindpainter K, Ganten D (Ed). The cardiac reninangiotensin system. Armonk (NY): Future Publishing Company, Inc 1994; 1-20.

2. Bell L, Luthringer DJ, Madri J, Warren CO. LTD. Autocrine angiotensin system regulation of bovino aortic endothelial cell migration and plasminogen activator involves modulation of proto-oncogene pp6ocsrc expression. J Clin Invest 1992; 89: 315-320.

3. Reddy MK, Baskaran K, Molteni A. Inhibitors of angiotensin-converting-enzyme Modulate mitosis and gene expression in pancreatic cancer cells (43942). Proc Soc Exp Biol Med 1995; 210: 221-226.

4. Senra A, Lopez Saez JB. Utility of serum activity of angiotensin converting enzyme as a tumor marker. Oncology 1993; 50: 430-435.

5. Wilkinson L. Systat: The system for statistic. Evanston, Il: Systat Inc 1990.

6. Gardner MJ, Altmam DG. Statistics with confidence. Confidence intervals and statistical guidelines. London: BMJ 1989.

7. Strike PW. Statistical methods in Laboratory Medicine. Oxford, Butterworth-Heinemann 1991.

8. Folkmam J. What in tumor dormancy: Can it be prolonged therapeuti- cally? David A. Karfnosky Memorial Lecture. Phyladelphia: ASCO Meeting 1996.

9. Robertson JIS, Ball SG. Hypertension. In Julian DG et al (Ed.). Diseases of the heart. London: Bailliere Tindall 1989; 1227-1292.

10. Frame KL, Patton K, Reed MJ, Gilchik MR, Parish DC. Angiotensinconverting enzyme and encephalinase in human breast cyst fluid. Br J Cancer 1996; 74: 807-813.

11. Goosens P, De Meester I, Vanhoof G, Scharpe S. Distribution of prolyl oligopeptidase in human peripheral tissues and body fluid. Eur J Clin Chem Clin Biochem 1996; 34: 17-22.

12. Franko AJ, Sharplin J, Ward WF, Taylor JM. Evidence for two patterns of inheritance of sensitivity to induction of lung fibrosis in mice by radiation, one of which involves two genes. Radiat Res 1996; 146: 68-74.

13. Poggi L, Renucci JF, Denolle T. Treatment of essential hypertension in general practice: An opon-label study of 47.351. French hypertension patients treated for one year with perindipril. Can J Cardiol 1994; 10: 121-124.

14. Palmeiro R, Garcia P, Senra A. Co-Morbility in cancer patients. Proccedings of the XIV International Cancer Congress. Budapest 1986; 3: 1010 . 